\title{
PSYCHOLOGICAL DETERMINANTS OF EMOTIONAL EATING: THE ROLE OF ATTACHMENT, PSYCHOPATHOLOGICAL SYMPTOM DISTRESS, LOVE ATTITUDES AND PERCEIVED HUNGER
}

\author{
Peta Stapleton and Eleanor Mackay \\ Bond University, (14 University Dr, Robina, QLD Australia, 4226), Australia
}

Received 2014-08-05; Revised 2014-08-12; Accepted 2014-09-13

\begin{abstract}
The present study aimed to investigate the psychological determinants of emotional eating in a national and international sample of healthy weight, overweight and obese adults $(N=226)$. Specifically, attachment styles, psychopathological symptom distress and love attitudes were explored for their ability to predict emotional eating. Findings supported the suggestion symptom distress may particularly predispose individuals to engaging in emotionally motivated overeating, with a large effect size observed. Preoccupied attachment was also a significant predictor of emotion eating, even after controlling for state-based inferences. However, there were no significant contributions of secure, fearful-avoidant, or dismissiveavoidant attachment style in the prediction of emotional eating. Similarly, none of the five primary love languages (e.g., words of affirmation, physical touch, receiving gifts and physical touch) accounted for a significant amount of variance in emotional eating. A mediating role of perceived hunger in the prediction of emotional eating from attachment-anxiety was also observed. Contributions and limitations of the present study, as well as recommendations for future research are also discussed.
\end{abstract}

Keywords: Emotional Eating, Attachment, Love Attitudes, Psychopathology

\section{INTRODUCTION}

Psychological determinants of emotional eating: The role of attachment, psychopathological symptom distress, love attitudes and perceived hunger

In 2008, the World Health Organisation (WHO) estimated $46 \%$ of the world's adult population over the age of 20 were obese or overweight (WHO, 2013), with this figure expected toincrease to $57.8 \%$ by 2030 (Kelly et al., 2008). Although efforts to address environmental factors/behaviours contributing to obesity are underway (Mitchell et al., 2011), research has highlighted the importance of addressing the internal determinants of dysfunctional eating behaviours in the prevention and treatment of obesity (Buckroyd and Rother, 2008).
An eating behaviour that has received particular attention as a risk factor for overeating and, therefore, obesity, is emotional eating. Psychological factors associated with emotional eating include: A preoccupied attachment disposition (Suldo and Sandberg, 2000), attachment-related anxiety (Scott et al., 2012), psychopathology (Goossens et al., 2009; Ouwens et al., 2009) and perceived hunger (Alexander and Siegel, 2013). However, these variables are yet to be collectively investigated.

\subsection{Emotional Eating}

Within literature the definition of emotional eating appears relatively consistent, commonly referred to as the tendency to consume food in response to negative or Corresponding Author: Peta Stapleton, Department of Psychology, Bond University, (14 University Dr, Robina, QLD Australia, 4226), Australia 
diffuse emotional states (Van Strien et al., 2005). From a biological perspective, eating in response to stress is viewed as irrational, as negative affect is associated with hormonal and gastric changes involved in appetite suppression (Blair et al., 1991). However, studies have consistently demonstrated biological, as well as psychological, factors contribute to an individual's motivation to consume food; however, in some cases, the latter can override the former (Dallman, 2010).

Despite the support for biologicalfood-mood effect, recent research (Macht, 2008) found improvements in experimentally induced negative mood states occurred only two minutes after eating chocolate, suggesting it was unlikely biological reactions alone were responsible for the effect of food on mood. Accordingly, some researchers have suggested the hedonic effects of certain foods may result, not only from nutritional or psychopharmacological properties, but also the association with socially and emotionally significant memories (Buckroyd and Rother, 2008; Goodspeed Grant, 2008). Thus, researchers have commonly characterised emotional eating as a complex, psychologically driven and dysfunctional coping mechanism, whereby eating can provide temporary comfort and relief from a preceding aversive experience (D'Arrigo, 2007).

According to psychosomatic theory, emotional eating occurs in response to aversive psychological and emotional states, such as fear and anxiety (Kaplan and Kaplan, 1957). Psychosomatic theory proposes feeding becomes associated with pleasurable situations, expressions of love and/or reward (instead of nutritional need) via early learning experiences (Stroebe et al., 2008). Consistently, the consumption of "comfort food" has been associated with improved emotional states across genders, ages and cultural backgrounds (Dubé et al., 2005). Over the years, numerous researchers have concluded emotional eating hinges on the recollection of mental associations, characterised by positive and intimate interpersonal experiences (Buckroyd and Rother, 2008; Dubé et al., 2005; Goodspeed Grant, 2008). These results uniquely align with psychosomatic theory, where eating in response to emotions is thought to offer reassurance and comfort, rather than distraction.

Qualitative research (Goodspeed Grant and Boersma, 2005; Goodspeed Grant, 2008) supports psychosomatic theory, with findings revealing emotional eaters often report difficult childhood family relationships, characterised by loneliness, a longing for interpersonal connectedness and a lack of self-care in adulthood. Frequently, this negative affect (or "emotional hunger") is similar to the experience of physical hunger when food deprived. As eating has strong connections with emotions of love and warmth, consumption of food can developinto a coping mechanism to manage experiences of emotional pain, by providing a sense of security and interpersonal closeness when family and social relationships are missingor difficult. However, few studies have explored the interaction between negative affect, hunger and emotional eating.

\subsection{Attachment and Emotional Eating}

Research suggests individuals who utilise food as a means of affect regulation may have marked psychological issues resultant from early childhood experiences, particularly difficult interpersonal relationships (Buckroyd and Rother, 2008). Attachment theory proposes every individual exhibits distinguishable differences in his/her disposition toward significant others and tendencies to seek strong interpersonal bonds. Bartholomew and Horowitz's (1991) four-category model of attachment is most often used: Secure, preoccupied, dismissive-avoidant and fearful-avoidant.

Individuals whose attachment pattern is predominantely secure tend to be characterised by asense of self-worth and more frequently engage in selfefficacious behaviour allowing them to effectively adjust to, or reduce, negative affect (Marsh, 2005). Conversely, dismissive-avoidant attachments have been associated with lower levels of engagement in external affect regulaton, with researchers suggesting this is due to a tendency to inhibit stress and repress emotional investment in problems (Mikulincer et al., 2003). Fearful-avoidant attachment has been associated with other overeating behaviours, such as binge eating (Pace et al., 2012) and other disordered eating symptoms (Suldo and Sandberg, 2000). Consequently, individuals with a fearful-avoidant attachment may be expected to have stronger emotional eating tendencies.

Similarly, individuals with a preoccupied attatchment (who typically lack self-worth, yet view others positively) have also been shown to be vulnerable to using passive and ruminative coping behaviours, such as drug abuse and food intake, to moderate emotional experiences (Mallinckrodt, 2010; Marsh, 2005). Numerous studies have supported this suggestion, with those exhibiting a preoccupied attachment reporting significantly higher levels of emotional and disordered eating (Suldo and Sandberg, 2000). Research has indicated individuals with preoccupied attachment styles are more likely to be obese,compared toindividuals with a dismissive-avoidant or secure attachment style. 
An important characteristic of attachment is the expectation it is a relatively stable trait, maintained throughout the lifespan (Bartholomew and Horowitz, 1991). However, research is increasingly acknowledging attachment constructs can be influenced by situational factors and people may also experience momentary fluctuations in their sense of security and/or insecurity. Emotional eating is often characterised by strong connections between food and socially significant memories and interpersonal difficulties can act as stressors triggering emotional eating. Although the dispositional approach to attachment emphasises these difficulties, it does not concentrate on state levels of attachment-related affect.

State attachment security refers to momentary perceptions about oneself and others, characterised by confidence, trust and self-reassurance (Gillath et al., 2009). Although the characteristics of state attachment appear similar to those of dispositional attachment styles, research has identified momentary shifts in senses of attachment are independent from a person's dispositional attachment style. Recently, Bezeau (2010) suggested the inclusion of state measures may provide a more thorough understanding of an individual's current attachment representations and how he/she relates to other constructs (such as emotional eating). However, the distinction between trait attachment styles and state levels of attachment have been largely neglected within literature.

\subsection{Psychological Symptom Distress and Emotional Eating}

Substantial research has suggested the negative affect associated with emotional eating is attachment-related. Giventhat interpersonal concerns are typical of the emotional eater, this research has irrefutably contributed to literature. However, there is substantial interindividual variation in the emotions responsible for triggering emotional eating (Macht, 2008). Thus, it appears our knowledge of the determinants of emotional eating could be expanded by investigating its relationship with broader measures of distress. In terms of specific distress, one study conducted by Konttinen et al. (2010) used the Center for Epidemiological Studies Depression Scale (CES-D) and the TFEQ emotional eating scores to explore the association between emotional eating and depressive symptoms. It was found CES-D scores were a positive predictor of emotional eating amongst a sample of 3714 normal weight, overweight and obese men and women.

Relative to other psychopathological symptoms, anxiety has also received substantial attention within emotional eating literature, although research is still somewhat scant. A study conducted by Goossens et al. (2009) revealed depression and anxiety scores were significant positive predictors of emotional eating after controlling for gender, age, BMI and socioeconomic status. However, this study was conducted amongst a sample of overweight children between the ages of eight and 18, therefore further research is needed to determine whether these results can be replicated in adult populations.

\subsection{Love Expression and Emotional Eating}

Within literature, there appears to be a consistent suggestion unsatisfying romantic relationships and attachment insecurity may serve as risk factors for emotional eating and food may function to satisfy an individual's emotional needs (Hernandez-Hons and Woolley, 2012). For example, Krause et al. (2000) revealed preliminary evidence suggesting social dependency and difficulties in expressing one's emotions were associated eating pathology, including food preoccupation. Qualitative studies have also reported emotional eaters tend to describe strong associations between emotions and food, with these emotions typically characterised by pleasure and comfort (Goodspeed Grant, 2008). Collectively, these results indicate individuals who experience interpersonal rejection or withdrawal may be predisposed to engaging in alternative behaviours (possibly emotional eating) to elicit affection and satisfy emotional needs. However, relational communication researchers have also established substantial inter-individual variabilities in peoples' communication patterns (Holtgraves, 1997). Therefore, the present study sought to utilise a novel approach of exploring whether individuals, who prefer to express and receive love in certain ways, are particularly vulnerable to using food for self-gratification.

Chapman (1992) love language typology is based on the metaphor of an emotional "love tank"; when a person feels emotionally close and loved by his/her significant other, his/her love tank is suggested to be full. Conversely, an individualperceiveshis/her emotional needs are unsatisfied, his/her love tank will be empty. However, according to Chapman (1992), people are not unanimous in what fills their love tank or, more specifically, what makes them feel emotionally satisfied. Hence, it is suggested people have preferred methods of emotional communication, which Chapman (1992) refers to as "love languages". Chapman (1992) identified five 
love languages: Words of affirmation (verbal compliments and statements of appreciation), quality time (focused attention and quality conversation), receiving gifts (tangible gifts and physical symbols of love), acts of service (doing favours for one another) and physical touch (from putting a hand on one's shoulder to intercourse).

\subsection{The Present Study}

There is limited research assessing whether dispositional attachment styles predict emotional eating above and beyond momentary fluctuations in a person's sense of attachment and other affective states unrelated to attachment. Psychopathological symptoms are also implicated in emotional eating, however, little research has been directed towards exploring the predictive value of symptom distress on emotional eating. Therefore, the present study also sought to investigate the relationship between distress and emotional eating. Perceived hunger may intervene in the relationship between attachment anxiety and emotional eating, therefore an additional aim of the current study was to examine whether this mediating relationship was observed when assessing state levels of attachment anxiety and to identify whether certain methods of emotional communication (i.e., love languages) were associated with emotional eating.

It was hypothesised that:

- Distress due to psychiatric symptomatology would be a significant positive predictor of emotional eating

- State levels of attachment anxiety would be a significant positive predictor of emotional eating whilst state levels of attachment avoidance and security will be significant negative predictors of emotional eating

- After controlling for symptom distress and state attachment-related inferences, preoccupied and fearful-avoidant attachment style would be a significant positive predictor of emotional eating while secure and dismissive-avoidant attachment styles would be significant negative predictors of emotional eating

- Perceived hunger would mediate the relationship between attachment anxiety and emotional eating. Specifically, attachment anxiety would significantly relate to higher levels of perceived hunger and higher levels of emotional eating

- A preference to express and receive love through physical touch love would be a significant positive predictor of emotional eating

\section{MATERIALS AND METHODS}

\subsection{Participants}

Healthy weight, overweight and obese adults were recruited through notices posted on online weight-related and general community noticeboards. Eligible participants were males and females over the age of 18 , currently in a romantic relationship, volunteering without incentive for participation. Choosing relationship status as an exclusion criterion enabled measurement of state attachment levels. Although 328 participants were initially recruited, the final sample consisted of 226 cases, after the exclusion of criteria and statistical violations.

Of the included participants, 197 were female (87.2\%) and 29 were male (12.8\%). Participants BMI ranged between 18.52 and 73.03 , with a mean of 27.44 $(S D=8.00)$. Of the participants, $49.6 \%$ had BMIs between 18.5 and 24.9 (classified as healthy), $22.1 \%$ had BMIs between 25 and 29.9 (classified as overweight) and $28.3 \%$ had BMIs greater than 30 (classified as obese). Additionally, 78 people were between 18 and 24 years old $(34.5 \%), 30$ people were between 25 and 29 years old $(13.3 \%), 27$ people were between 30 and 35 years old $(11.9 \%), 15$ people were between 36 and 39 years old $(6.6 \%), 17$ people were between 40 and 45 years old $(7.5 \%), 11$ people were between 46 and 49 years old $(4.9 \%)$ and 48 people were over 50 years old $(21.2 \%)$.

Approximately $69 \%$ of the sample reported they were born in Australia, 9.7\% reported the USA, 8.8\% reported England, whilst the remaining $12.5 \%$ reported they were born in other countries (e.g., New Zealand, Scotland and India). Almost half the sample (40.3\%) had completed a Bachelor's degree, whilst $19.9 \%$ had only completed high school or equivalent, 9.3\% had completed vocational or technical training, $17.7 \%$ had completed a Master's degree and $4 \%$ had completed a Doctoral degree. The remaining 20 participants identified their highest level of education completed "other" $(8.8 \%)$.

\subsection{Demographic Questions}

For the purpose of describing the sample, participants were asked to provide information regarding age, gender, marital status, number of people in their household, country of birth and highest level of completed education.

\subsection{Anthropometric Measures}

Participants were asked to provide their current height and weight measurements for the purpose of estimating BMI, calculated as weight in kilograms 
divided by height in meters squared $\left(\mathrm{kg} / \mathrm{m}^{2}\right)$. Recent research suggests self-reported anthropometric measurements accurately quantify the body size of most middle-aged, elderly and young individuals' ( $\mathrm{Ng}$ et al., 2011). Thus, each participant's BMI was yielded in the present study to classify participants as underweight $(<18.5)$, healthy weight (18.5 to 24.9$)$, overweight (25.0 to 29.9), or obese $(\geq 30.0)$.

\subsection{Three Factor Eating Questionnaire (TFEQ; Stunkard and Messick, 1985)}

The TFEQ is a 51-item self-report instrument designed to examine three cognitive and behavioural aspects of eating behaviour: Cognitive restraint, disinhibition of eating and perceived hunger. Using multitrait/multi-item analysis Karlsson et al. (2000), identified an alternative scoring scheme where three of the original items can reliably measure emotional eating. As this study formed part of a broader research project, the TFEQ was administered in its entirety; however, only items addressing perceived hunger and emotional eating were relevant to the present analysis. Previous research has found TFEQ to be a valid and psychometrically sound instrument, with the perceived hunger scale and emotional eating scale demonstrating good internal consistencies $(\alpha=0.80$ and 0.85 , respectively) (Karlsson et al., 2000). Results of the current study revealed internal consistencies of 0.82 for both subscales.

\subsection{Relationships Questionnaire Bartholomew and Horowitz, 1991)}

(RQ;

The RQ is a 5-item self-report questionnaire designed to assess dispositional attachment styles: Secure, preoccupied, fearful-avoidant and dismissive-avoidant. The RQ consists of four brief vignettes, each describing one of the aforementioned attachment styles. Respondents were initially asked to identify the single attachment pattern best describing them, with this forced-choice paragraph presented first to minimise order effects. However, Bartholomew and Horowitz (1991) recommend a dimensional approach to assessment. Therefore, respondents were also asked to indicate using a 7-point Likert scale $(1=$ disagree strongly to $7=$ agree strongly), the degree to which each description corresponded with their attitude toward relationships in general. Thus, higher scores reflected an increased tendency toward a particular attachment style. Previous research has found the RQ to be a valid and psychometrically sound instrument, with evidence suggesting good temporal stability over an eight- month period $(r=0.72$ to 0.96$)$ (Scharfe and Bartholomew, 1994).

\subsection{State Adult Attachment Measure (SAAM; Gillath et al., 2009)}

The SAAM is a 21-item self-report questionnaire designed to measure state levels of attachment-related security, anxiety and avoidance. Each domain was assessed by seven items and participants were asked to rate the degree to which each statement (e.g., I wish someone would tell me they really love me), reflected their current feelings using a 7-point Likert scale $(1=$ disagree strongly to $7=$ agree strongly). Scale scores were yielded by summing response values corresponding to that domain, with higher scores indicative of higher security, anxiety, or avoidance. Internal consistency coefficients of $0.83,0.84$ and 0.87 for the avoidance, anxiety and security subscales, respectively, have also been reported (Gillath et al., 2009). In the present study, Cronbach' salphas were similar: 0.81 for avoidance, 0.87 for anxiety and 0.88 for the security scales.

\subsection{Symptom Assessment-45 Questionnaire (SA-45; Strategic Advantages, 2000)}

The SA-45 is a 45-item questionnaire designed to assess levels of psychological distress due to psychiatric symptomatology. Respondents were required to rate the degree to which a problem (e.g., feeling lonely) has bothered or distressed them over the past week using a 5point Likert scale $(1=$ not at all to $5=$ extremely $)$. For the purpose of the current study, the Positive Symptom Total (PST) was used to indicate the degree of distress present and was yielded by summing the total number of items with a response other than "not at all". PST scores ranged from zero to 45, with higher scores indicating a greater level of distress. Research has found the SA-45 to be a valid and psychometrically sound instrument, with good internal consistencies ranging from 0.71 to 0.87 , with the current study revealing an internal consistency of 0.94 for the PST.

\subsection{Five Love Languages Questionnaire (FLLQ; Chapman, 1992)}

The FLLQ is a profiling system designed to identify an individuals' preferred mode of emotional communication. The instrument examines five emotional communication styles specific to romantic relationships: Words of affirmation, acts of service, receiving gifts, quality time and physical touch. The questionnaire consists of 30 statement pairs, for instance "I like to 
spend time with my partner" and "I like to receive little gifts from my partner", with participants required to identify the statement they feel best describes their desires in their current relationship. For each style of emotional communication 12 statements are given and categorical assessment of each participant's love language is based on the communication style most frequently endorsed. Although Chapman (1992) proposed individuals have primary love languages, it was also noted most people respond to each method of love expression to varying degrees and, therefore, a dimensional scoring approach was taken, similar to the measurement of attachment.

Empirical data examining the FLLQ is currently limited. However, research (Egbert and Polk, 2006) has supported the five-factor solution, whilst a validated measure of relational maintenance behaviours (i.e., the Relational Maintenance Behaviours scale), has been shown to significantly predict each of the love languages in the expected direction. Using a modified response format (i.e., 4-point Likert scale) each of the love languages has also been shown to be reliable amongst undergraduate students in current romantic relationships, with internal consistencies ranging between 0.77 and 0.85 (Egbert and Polk, 2006).

\subsection{Procedure}

Ethical approval was obtained from the University Human Research Ethics Committee. Participants were recruited from national and international weightrelated (e.g., Obesity Help) or general community noticeboards (e.g., Nnub). Potential participants were provided with an online link directing them to an explanatory statement and consent form on a secure web-based system (Psych Data).

\section{RESULTS}

The data were analysed using SPSS version 22. An alpha level of .05 was utilised to determine the statistical significance of all results.

\subsection{Preliminary Analysis}

A series of preliminary analyses were conducted using chi-square analyses and one-way between-groups Analysis Of Variance (ANOVA) to determine whether the demographic variables (gender, age, highest level of completed education and marital status) covaried with any of the dependent or independent variables (RQ, SAAM, SA-45, TFEQ emotional eating and FLLQ scores). Chi-square analyses revealed significant differences between the three weight category groups (e.g., normal weight Vs overweight Vs obese) on gender $\mathrm{x}^{2}(2)=8.35, p=0.015$, age $\mathrm{x}^{2}(12)=52.70, p=<0.001$ and education $\mathrm{x}^{2}(10)=28.45, p=0.002$.

Results revealed gender significantly influenced RQ dismissive-avoidant $F(1,224)=7.653, p=0.006$ and TFEQ emotional eating $F(1,224)=15.563, p<0.001$. Additionally, age significantly impacted RQ fearfulavoidant $F(6,219)=2.37, p=0.031$ and SAAM attachment-anxiety $F(6,219)=4.23, p<0.001$. No significant differences were observed for marital status or education, indicating these variables did not significantly impact any of the predictor or criterion variables.

To determine whether BMI significantly covaried with any previously mentioned variables, bivariate correlations were yielded. This analysis revealed BMI was significantly positively associated with TFEQ emotional eating $(r=0.371)$. It was also identified BMI was significantly negatively associated with RQ secure attachment $(r=-0.252)$ and SAAM attachmentsecurity $(r=-0.182)$ scores.

\subsection{Main Analyses}

Psychopathology, Attachment Style and State Attachment, as a Predictor of Emotional Eating. To assess the predictive value of attachment styles, psychopathological symptom distress and state attachment levels, a Hierarchical Multiple Regression (HMR) analysis was conducted on emotional eating scores. As age, gender and BMI were identified as significantly impacting at least one of the criterion or predictor variables in the preliminary analyses, these were controlled for by entering them at step one. SA45 PST scores were entered at step two, SAAM scores were entered at step three and RQ scores were entered at step four. Results of the HMR predicting emotional eating scores from SA-45 PST, SAAM and RQ scores, after controlling for gender, age and BMI, are presented in Table 1.

On step one, gender, age and BMI accounted for a significant $20 \%$ of variance in emotional eating, adjusted $R^{2}=0.20, F(8,217)=8.01, p<0.001$. As seen in Table 1, the coefficients indicated BMI was the strongest predictor of emotional eating, where a higher BMI significantly predicted higher emotional eating scores. Gender was also a significant predictor of emotional eating, where women reported higher levels of emotional eating than men. Age did not significantly predict emotional eating, except for between the ages of 46 to 49 where emotional eating scores were significantly higher than participants between the ages of 18 and 24 . 
Table 1. Hierarchical Multiple Regression Analysis Predicting Emotional Eating from SA-45 PST, SAAM Anxiety, Security and Avoidance and RQ Secure, Fearful-Avoidant, Preoccupied and Dismissive-Avoidant Scores, After Controlling for Gender, Age and BMI

\begin{tabular}{|c|c|c|c|c|c|c|}
\hline Predictor & $\mathrm{R}$ & $\mathrm{R}^{2}$ & $\mathrm{~B}$ & SE B & $\beta$ & $95 \% \mathrm{CI}$ for $\mathrm{B}$ \\
\hline Step 1 & $0.48^{* * *}$ & 0.23 & & & & \\
\hline Constant & & & $-0.87 *$ & 0.34 & & {$[-1.53,-0.21]$} \\
\hline Gender & & & $10.00 * * *$ & 0.23 & 0.26 & {$[0.54,1.45]$} \\
\hline 25 to 29 years old & & & 0.35 & 0.25 & 0.09 & {$[-0.15,0.84]$} \\
\hline 30 to 25 years old & & & -0.26 & 0.27 & -0.07 & {$[-0.79,0.26]$} \\
\hline 36 to 39 years old & & & -0.07 & 0.33 & -0.01 & {$[-0.72,0.59]$} \\
\hline 40 to 45 years old & & & -0.18 & 0.33 & -0.04 & {$[-0.82,0.46]$} \\
\hline 46 to 49 years old & & & $0.88^{*}$ & 0.38 & 0.15 & {$[0.13,1.63]$} \\
\hline Over 50 years old & & & -0.00 & 0.23 & 0.00 & {$[-0.45,0.45]$} \\
\hline BMI & & & $0.06 * * *$ & 0.01 & 0.36 & {$[0.04,0.07]$} \\
\hline Step 2 & $0.54 * * *$ & 0.30 & & & & \\
\hline Constant & & & $-1.28 * * *$ & 0.33 & & {$[-1.94,-0.63]$} \\
\hline PST & & & $0.03 * * *$ & 0.01 & 0.27 & {$[0.02,0.05]$} \\
\hline Step 3 & $0.54 * * *$ & 0.30 & & & & \\
\hline Constant & & & -1.59 & 0.87 & & {$[-3.30,0.11]$} \\
\hline Anxiety & & & 0.00 & 0.01 & 0.02 & {$[-0.01,0.02]$} \\
\hline Security & & & 0.00 & 0.01 & 0.02 & {$[-0.02,0.03]$} \\
\hline Avoidance & & & 0.00 & 0.01 & 0.01 & {$[-0.03,0.03]$} \\
\hline Step 4 & $0.58 * * *$ & 0.34 & & & & \\
\hline Constant & & & -1.35 & 0.96 & & {$[-3.24,0.53]$} \\
\hline Secure & & & -0.05 & 0.05 & -0.08 & {$[-.15,0.04]$} \\
\hline Fearful & & & 0.00 & 0.05 & 0.01 & {$[-.09,0.10]$} \\
\hline Preoccupied & & & $0.12 * * *$ & 0.05 & 0.18 & {$[0.03,0.21]$} \\
\hline Dismissive & & & -0.09 & 0.05 & -0.12 & {$[-0.18,0.00]$} \\
\hline
\end{tabular}

Note: SA-45 = Symptom Assessment-45 Questionnaire; PST = Positive Symptom Total; SAAM = State Adult Attachment Measure; $\mathrm{RQ}=$ Relationship Questionnaire; BMI = Body Mass Index.

$* \mathrm{p}<0.05, * * \mathrm{p}=0.01, * * * \mathrm{p}<0.001$

On step two of the HMR analysis, PST scores were added in to the regression equation and accounted for an additional $7 \%$ of variance in emotional eating scores $R_{\text {change }}^{2}=0.07, F_{\text {change }}(1,216)=20.46, p<0.001$. Together, the predictor variables at step two explained $27 \%$ of variance in emotional eating scores, adjusted $R^{2}$ $=0.27, F(9,216)=10.03, p<0.001$. By Cohen's (1988) standards, a combined effect of this magnitude can be considered "large" $\left(f^{2}=0.36\right)$. Further analysis of the coefficients indicated gender, BMI and the 46 to 49 year old age group remained significant predictors in the second step. BMI was identified as the strongest predictor of emotional eating and PST scores were the second strongest predictor, where higher PST scores predicted significantly higher emotional eating scores.

On step three of HMR, SAAM scores were added in to the regression equation and, collectively, the predictor variables at step three explained a significant $26 \%$ of variance in emotional eating scores, adjusted $R^{2}=0.26$, $F(12,213)=7.44, p<0.001$. By Cohen's (1988) standards, a combined effect of this magnitude can be considered "medium" $\left(f^{2}=0.34\right)$. Analysis of the change statistics, however, indicated SAAM scores did not account for a significant amount of variance beyond that already accounted for by gender, age and BMI and SA45 PST scores, $F_{\text {change }}(3,213)=0.07, p=0.977$. Analysis of the coefficients at step three indicated only gender, the 46 and 49 years category, BMI and SA-45 PST scores were significant predictors of emotional eating in the second step.

On step four of the analysis, RQ scores were added in to the regression equation and accounted for an additional $4 \%$ of the variance in emotional eating scores, $R_{\text {change }}^{2}=0.04, F_{\text {change }}(4,209)=3.35, p=0.011$. Together, the predictor variables at step four explained $29 \%$ of the variance in emotional eating scores, adjusted $R^{2}=0.29, F(16,209)=6.67, p<0.001$. By Cohen's (1988) standards, a combined effect of this magnitude can be considered "large" $\left(f^{2}=0.403\right)$. Further analysis of the coefficients indicated gender, BMI and SA-45 PST scores, but not being between 46 to 49 years old, remained significant predictors in the fourth and final step of the regression. The standardised coefficient betas also indicated only preoccupied attachment style scores 
significantly predicted emotional eating, where higher levels of preoccupied attachment predicted higher emotional eating scores. As demonstrated in Table 1, no other attachment styles significantly predicted emotional eating scores, although dismissive-avoidant attachment approached significance.

\subsection{Perceived Hunger as a Mediator between Attachment Anxiety and Emotional Eating}

Although SAAM attachment anxiety was not identified as a significant predictor in of emotional eating, it was possible stronger predictors (e.g., SA-45 PST scores) masked the relationship between attachment-anxiety and emotional eating. To investigate the possible mediating effect of perceived hunger in the prediction of emotional eating scores from attachment anxiety, mediation analyses were conducted.

Attachment anxiety scores accounted for a significant $2 \%$ of the variability in emotional eating scores, adj $R^{2}=$ $0.02, F(1,224)=4.79, p=0.030$. Examination of the coefficients indicated higher attachment anxiety scores predicted higher emotional eating scores $(B=0.02, S E B$ $=0.01, \beta=0.15, p=0.030)$. Attachment anxiety scores also accounted for a significant $3 \%$ of the variability in perceived hunger scores, $\operatorname{adj} R^{2}=0.03, F(1,224)=8.06$, $p=0.005$. Examination of the coefficients indicated higher attachment anxiety scores predicted higher emotional eating scores $(B=0.07, S E B=0.03, \beta=0.19$, $p=0.05)$. Perceived hunger accounted for a significant $19 \%$ of the variability in emotional eating scores, adjusted $R^{2}=0.19, F(1,224)=52.79, p<0.001$ and analysis of the coefficient indicated higher perceived hunger scores predicted higher emotional eating scores $(B=0.15, S E B=0.02, \beta=0.44, p<0.001)$.

As can be seen in Fig. 1, the standardised regression coefficient between attachment anxiety and emotional eating decreased when perceived hunger scores were added into the model (a shift of $\beta=0.15$ to $\beta=0.07$ ). Perceived hunger scores were found to reduce the significance of the standardised regression coefficient between attachment anxiety and emotional eating (a shift of $p=0.030$ to $p=0.285$ ), suggesting a full mediation of attachment anxiety of scores by perceived hunger scores, in the prediction of emotional eating scores. Figure 1 shows the mediating relationship of perceived hunger scores between attachment anxiety and emotional eating scores.

As can be seen in Fig. 1, the addition of perceived hunger weakened the strength of the predictive relationship between attachment-anxiety and emotional eating. To determine whether the observed change was significant, a Sobel test was conducted (Sobel, 1982). This analysis indicated the change in the predictive value of attachment-anxiety was significant, $\mathrm{Z}=2.68, p=$ 0.007 . Thus, perceived hunger fully mediated the predictive relationship between attachment-anxiety and emotional eating scores.

\subsection{Love Languages as Predictors of Emotional Eating}

To investigate the predictive value of love languages for emotional eating, a HMR analysis was conducted on emotional eating total scores. As gender and BMI were identified as covarying with the criterion variable, these variables were entered at step one. FLLQ scores were subsequently entered at step two to determine whether they accounted for a significant amount of variance above and beyond that already accounted for by the demographic variables. Results of the HMR predicting emotional eating scores from FLLQ scores are presented in Table 2.

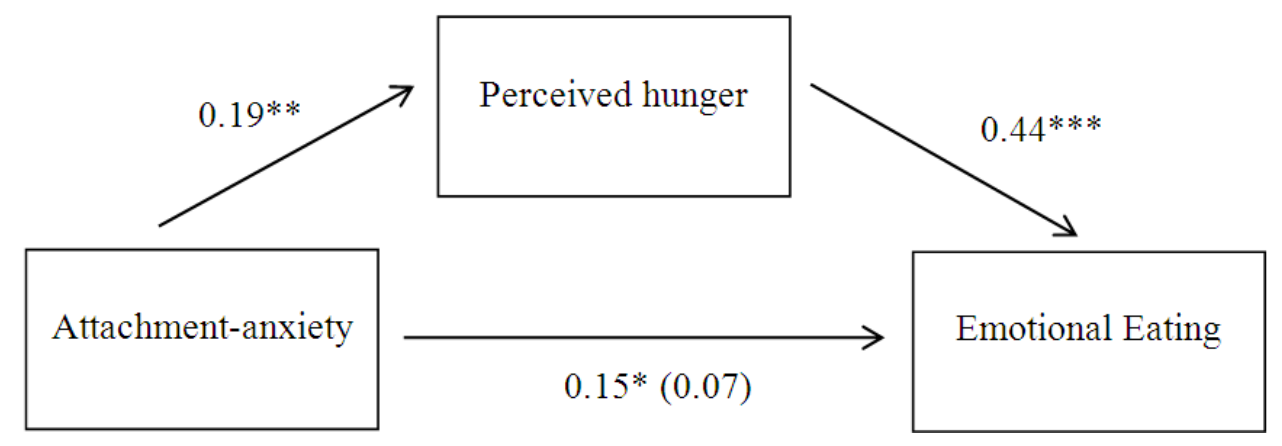

Fig. 1. The relationship between attachment-anxiety and emotional eating scores, with perceived hunger as a mediator. Note. Values represent the standardised $\beta$ weights. $\beta$ in the parenthesis indicates the changed value after perceived hunger was added in to the regression equation 
Peta Stapleton and Eleanor Mackay / Current Research in Psychology 5 (2): 77-88, 2014

Table 2. Hierarchical Multiple Regression Analysis Predicting Emotional Eating from FLLQ Quality Time, Gifts, Physical Touch and Acts of Service Scores, After Controlling for BMI and Gender

\begin{tabular}{|c|c|c|c|c|c|c|}
\hline Predictor & $\mathrm{R}$ & $\mathrm{R}^{2}$ & $\mathrm{~B}$ & SE B & $B$ & $95 \% \mathrm{CI}$ for $\mathrm{B}$ \\
\hline Step 1 & $0.44 * * *$ & 0.19 & & & & \\
\hline Constant & & & -0.74 & 0.33 & & {$[-1.40,-0.08]$} \\
\hline BMI & & & $0.06 * * *$ & 0.01 & 0.35 & {$[0.04,0.08]$} \\
\hline Gender & & & $0.90 * * *$ & 0.23 & 0.24 & {$[0.45,1.36]$} \\
\hline Step 2 & $0.48 * * *$ & 0.23 & & & & \\
\hline Constant & & & -0.88 & 1.00 & & {$[-2.85,1.09]$} \\
\hline Quality Time & & & -0.06 & 0.05 & -0.10 & {$[-0.16,0.04]$} \\
\hline Receiving Gifts & & & -0.01 & 0.05 & -0.02 & {$[-0.11,0.09]$} \\
\hline Physical Touch & & & 0.04 & 0.04 & 0.09 & {$[-0.03,0.11]$} \\
\hline Acts of Service & & & 0.06 & 0.05 & 0.09 & {$[-0.03,0.16]$} \\
\hline
\end{tabular}

Note: TFEQ $=$ Three Factor Eating Questionnaire. FLLQ = Five Love Languages Questionnaire; BMI = Body Mass Index ${ }^{*} \mathrm{p}<0.05,{ }^{*} \mathrm{p}=0.01, * * * \mathrm{p}<0.001$

On step one of the HMR, gender and BMI accounted for a significant $18 \%$ of variance in emotional eating scores, adjusted $R^{2}=0.18, F(2,223)=26.19, p<0.001$, with BMI the strongest positive predictor of emotional eating at this step (i.e., higher BMIs predicted significantly greater emotional eating scores). Additionally, gender was a significant predictor of emotional eating, with women scored higher than men.

On step two of the HMR, FLLQ words of affirmation, quality time, receiving gifts and physical touch total scores were added to the regression equation and accounted for an additional $4 \%$ of the variance in emotional eating scores, $R_{\text {change }}^{2}=0.04, F_{\text {change }}(4,219)=$ 2.48, $p=0.045$. Collectively, the predictor variables at step two explained $20 \%$ of the variance in emotional eating scores, adjusted $R^{2}=0.20, F(6,219)=10.61$, $p<0.001$. According to Cohen's (1988) guidelines, a combined effect of this magnitude can be considered "medium" $\left(f^{2}=0.26\right)$. Further examination of the coefficients, however, indicated none of the love language individually accounted for a significant amount of variance in emotional eating scores.

\section{DISCUSSION}

Research has identified the missing component in weight loss attempts is the failure to address underlying psychological factors that contribute to overeating (Goodspeed Grant, 2008). The current study investigated the psychological determinants of emotional eating in a national and international sample of healthy weight, overweight and obese participants.

\subsection{Predicting Emotional Eating from Psychological Symptom Distress}

Previous studies indicate an association between psychopathological disturbance and emotional eating
(Goossens et al., 2009; Ouwens et al., 2009). However, research regarding this relationship remains somewhat limited. Consistent with hypotheses, higher levels of disturbance due to psychopathological symptoms significantly predicted higher emotional eating scores. As the effect size for PST scores was considered large, these results support the argument emotional eaters may have underlying psychological issues contributing to his/her eating behaviour.

Although research has demonstrated childhood traumas (e.g., emotional and/or physical neglect) are frequently reported in eating disordered populations, researchers (Kong and Bernstein, 2008) suggest negative psychological affect, rather than the traumatic experience itself, may be key to emotional eating tendencies. Thus it appears when the psychological consequences of difficult experiences are unresolved and persist into adulthood, these experiences may predispose individuals to eating pathologies (Kong and Bernstein, 2008). This interpretation aligns with explanations of attachment insecurity, where psychological histories are implicated in the association between attachment and emotional eating. Overall results of the present study indicate psychopathological symptoms are an important negative affective experience that may trigger emotional eating.

\subsection{Predicting Emotional Eating from Attachment}

Attachment styles were examined in the present study to investigate the association between emotional eating and disturbances in the early development of representations about interpersonal relationships. Inconsistent with expectations, secure, dismissiveavoidant and fearful-avoidant attachment did not significantly predict emotional eating in the present study. Whilst this finding is not surprising for fearful- 
avoidant attachment style given discrepancies within current literature (Pace et al., 2012), the results regarding secure and dismissive-avoidant attachment are surprising given the inconsistency with current literature.

Secure attachment style has been associated with active and constructive coping mechanisms that enhance adaptive self-regulation skills, which are important for managing stress (Mikulincer et al., 2003). The dismissal of affective information, characteristic of dismissiveavoidant attachment style, has also been associated with suppressed emotional experiences (Mikulincer et al., 2003). Theoretically, these attachment styles should, therefore, lead to lower levels of dependence on maladaptive regulatory behaviours, such as emotional eating. Whilst results of the present study may suggest state-related negative affect is a more important predictor of emotional eating than attachment style, it should also be noted participants were recruited from weight-related online noticeboards where people tend to seek interpersonal support to manage overeating.

\subsection{Perceived Hunger as a Mediator between Attachment-Anxiety and Emotional Eating}

Difficulties in interpersonal functioning can lead to social stress, consequently increasing one's vulnerability to emotional overeating (Scott et al., 2012). Hence, it seems plausible attachment-anxiety may be an important predictor of emotional eating, which has been supported in previous empirical literature. Whilst the present study hypothesised attachment-related anxiety would be a significant positive predictor of emotional eating scores, psychosomatic theory suggests people will only eat in response to negative affect when they simultaneously misperceive themselves as hungry. Consistent with psychosomatic theory and previous research, perceived hunger fully mediated the relationship between attachment-related anxiety and emotional eating, indicating negative affect may be associated with emotional eating via the misperception of the physical sensations of emotional arousal for hunger signals. This finding is particularly important in managing emotional eating, as it may suggest whether or not people experiencing attachment-related anxiety perceive themselves as hungry is a more important predictor of emotional eating than the aversive emotional experience itself.

\subsection{Predicting Emotional Eating from Love Languages}

Research has suggested eliciting comfort and satisfying emotional needs may positively reinforce emotional eating, however there is substantial interindividual variation in the way people prefer to express and receive love (Krause et al., 2000). Love languages were examined in the present study to determine whether certain methods of emotional communication significantly predicted emotional eating. Specifically, it was hypothesised the love language of physical touch would be a significant positive predictor of emotional eating. However, this hypothesis was not supported. Although no preliminary hypotheses were made regarding Chapman (1992) remaining four languages of love, no significant relationship between these methods of relational communication and emotional eating were observed.

Although results indicate methods of emotional communication may not play an important role in the prediction of emotional eating, an apparent need to identify methods of satisfying peoples' emotional cravings remains. Therefore, future experimental research should clarify whether an intervention based on Chapman (1992) love languages can be used to reduce emotional eating symptoms by enhancing relational satisfaction and reducing attachment-related stressors.

\subsection{Limitations and Recommendations for Future Research}

The present study had several limitations. First, the causal direction of the observed relationships cannot be confidently determined from the data. For instance, the results identified that psychopathological symptomatology accounted for a significant amount of variance in emotional eating scores, but it remains unclear if distress symptoms are a cause or consequence of emotional eating. Several sampling limitations are also noted. For example, participants were recruited from online weight forums, therefore it possible the sample was not representative of the broader population in terms of psychological characteristics. According to Evers et al. (2011), self-report emotional eating scales should be conceptualized as measuring an individual's attitude toward emotions and eating, rather than an indicator of actual food intake. Therefore, experimental replication of the current study, with an objective measure of food consumption in response to negative affect, would be beneficial to determine whether results of the current study can generalize to actual eating behave our.

Literature has suggested standard weight-loss programs typically fail to achieve long-termsuccess and this has largely been attributed to the failure of interventions to address psychological determinants of overeating behaviours. Results of the current study revealed a large effect between symptom distress and emotional eating, indicating clinicians may benefit from direct treatment 
strategies towards managing symptom distress. Findings of the current study also provide important insight into the robustness of the relationship between preoccupied attachment and emotional eating and highlight the importance of considering how psychological issues affecting an emotional eater may relate to his/her history.

\section{CONCLUSION}

However, the present study also demonstrated perceived hunger significantly mediated the relationship between attachment-anxiety and emotional eating. Although this aligns with psychosomatic theory, the implications of this finding should be considered preliminary in nature. Nonetheless, this mediating effect indicates strategies for accurately distinguishing physiological sensations of emotional arousal from hunger may be critical in the management of emotional eating. Additionally, the development of adaptive ways to meet emotional hunger may also be functional for reducing reliance on food for basic needs satisfaction.

\section{FUNDING INFORMATION}

The authors have no support or funding to report.

\subsection{Author's Contributions}

All authors equally contributed in this study.

\subsection{Ethics}

This article is original and contains unpublished material. The corresponding author confirms that all of the other authors have read and approved the manuscript and no ethical issues involved.

\section{REFERENCES}

Alexander, K.E. and H.I. Siegel, 2013. Perceived hunger mediates the relationship between attachment anxiety and emotional eating. Eating Behaviours, 14: 374-377. DOI: 10.1016/j.eatbeh.2013.02.005

Bartholomew, K. and L. Horowitz, 1991. Attachment styles among young adults: A test of a four-category model. J. Personality Social Psychol., 61: 226-244. DOI: $10.1037 / 0022-3514.61 .2 .226$

Bezeau, C., 2010. From the cradle to the workplace: Attachment and the reported provision of need support (Doctoral dissertation).

Blair, E.H., R.R. Wing and A. Wald, 1991. The effects of laboratory stressors on glycemic control and gastrointestinal transit-time. Psychosomatic Medicine, 53: 133-143. DOI: 10.1016/j.tem.2009.10.004
Buckroyd, J. and S. Rother, 2008. Psychological Responses to Eating Disorders and Obesity: Recent and Innovative Work. 1st Edn., John Wiley and Sons, Hoboken, ISBN-10: 0470061642, pp: 191.

Chapman, G., 1992. The 5 Love Languages: The Secret to Love that Lasts. 1st Edn., Northfield Publishing, Chicago.

Dallman, M.F., 2010. Stress-induced obesity and the emotional nervous system. Trends Endocrinol. Metabolism, 3: 159-165. DOI: $10.1016 /$ j.tem.2009.10.004

D'Arrigo, T., 2007. Emotional eating. A sneak attack on weight loss. Diabetes Forecast. PMID: 17707992

Dubé, L., J.L. LeBel and J. Lu, 2005. Affect asymmetry and comfort food consumption. Physiol. Behaviour, 86: 559-567. DOI: 10.1016/j.physbeh.2005.08.023

Egbert, N. and D. Polk, 2006. Speaking the language of relational maintenance: A validity test of Chapman's (1992) five love languages. Commun. Res. Reports, 23: 19-26. DOI: 10.1080/17464090500535822

Evers, C., D.T. de Ridder and M.A. Adriaanse, 2011. Assessing yourself as an emotional eater: Mission impossible? Appetite, 57: 536-536. DOI: 10.1016/j.appet.2011.05.005

Gillath, O., J. Hart, E.E. Noftle and G.D. Stockdale, 2009. Development and validation of a state adult attachment measure. J. Res. Personality, 43: 362-373. DOI: 0.1016/j.jrp.2008.12.009

Goodspeed Grant, P., 2008. Food for the Soul: Social and Emotional Origins of Comfort Eating in the Morbidly Obese in Psychological Responses to Eating Disorders and Obesity. London, England: Wiley and Sons.

Goodspeed Grant, P. and H. Boersma, 2005. Making sense of being fat: A hermeneutic analysis of adults' explanations for obesity. Counsell. Psychotherapy Res. Linking Res. Practice, 5: 212-220. DOI: $10.1080 / 17441690500310429$

Goossens, L., C. Braet, L. Van Vlierberghe and S. Mels, 2009. Loss of control over eating in overweight youngsters: The role of anxiety, depression and emotional eating. Eur. Eating Disorders Rev., 17: 68-78. DOI: $10.1002 /$ erv.892

Hernandez-Hons, A. and S.R. Woolley, 2012. Women's experiences with emotional eating and related attachment and sociocultural processes. J. Marital Family Therapy, 38: 589-603.

DOI: $10.1111 /$ j.1752-0606.2011.00239

Holtgraves, T., 1997. Styles of language use: Individual and cultural variability in conversational indirectness. J. Personality Social Psychol., 73: 624-637. DOI: 10.1037/0022-3514.73.3.624 
Kaplan, H.I. and H.S. Kaplan, 1957. The psychosomatic concept of obesity. J. Nervous Mental Disease, 125: 181-201. PMID: 13481715

Karlsson, J., L. Persson, L. Sjöström and M. Sullivan, 2000. Psychometric properties and factor structure of the Three-Factor Eating Questionnaire (TFEQ) in obese men and women. Results from the Swedish Obese Study (SOS) study. Int. J. Obesity, 24: 1715-1725. DOI: $10.1038 /$ sj.ijo.0801442

Kelly, T., W. Yang, C.S. Chen, K. Reynolds and J. He, 2008. Global burden of obesity in 2005 and projections to 2030. Int. J. Obesity, 32: 1431-1437. DOI: $10.1038 /$ ijo.2008.102

Kong, S. and K. Bernstein, 2008. Childhood trauma as a predictor of eating psychopathology and its mediating variables in patients with eating disorders. J. Clin. Nurs., 18: 1897-1907.

DOI: $10.1111 /$ j.1365-2702.2008.02740

Konttinen, H., S. Mannisto, S. Sarlio-Lahteenkorva, K. Silventoinen and A. Haukkala, 2010. Emotional eating, depressive symptoms and self-reported food consumption: A population-based study. Appetite, 54: 437-479. DOI: 10.1016/j.appet.2010.01.014

Krause, E.D., C.I. Robins and T.R. Lynch, 2000. A meditational model relating sociotropy, ambivalence over emotional expression and disordered eating. Psychol. Women Q., 24: 328-335. DOI: $10.1111 / \mathrm{j} .1471-6402.2000 . t b 00215$

Macht, M., 2008. How emotions affect eating: A fiveway model. Appetite, 1: 1-11.

DOI: 10.1016/j.appet.2007.07.002

Mallinckrodt, B., 2010. Attachment, social competencies, social support and interpersonal process in psychotherapy. Psychotherapy Res., 10: 239-266. DOI: 10.1093/ptr/10.3.239

Marsh, G.M., 2005. The role of attachment to obesity and psychopathology. University of Plymouth.

Mikulincer, M., P.R. Shaver and D. Pereg, 2003. Attachment theory and affect regulation: The dynamics, development and cognitive consequences of attachment-related strategies. Motivation Emot., 27: 77-102. DOI: 10.1023/A: 1024515519160

Mitchell, N.S., V.A. Catenacci, H.R. Wyatt and J.O. Hill, 2011. Obesity: Overview of an epidemic. Psychiatric Clin. North Am., 34: 717-732. DOI: $10.1016 /$ j.psc.2011.08.005

Ng, S.P., R. Korda, M. Clements, I. Latz and A. Bauman et al., 2011. Validity of self-reported height and weight and derived body mass index in middle-aged and elderly individuals in Australia. Aus. New Zealand J. Public Health, 35: 557-563. DOI: $10.1111 /$ j. 1753-6405.2011.00742
Ouwens, M.A., T. Van Strien and J.F.J. Van Leeuwe, 2009. Possible pathways between depression, emotional and external eating: A structural equation model. Appetite, 53: 245-248. DOI: 10.1016/j.appet.2009.06.001

Pace, U., M. Cacioppo and A. Schimmenti, 2012. The moderating role of father's care on the onset of binge eating symptoms among female late adolescents with insecure attachment. Child Psychiatry Human Develop., 43: 282-292.

DOI: $10.1007 / \mathrm{s} 10578-011-0269-7$

Scharfe, E. and K. Bartholomew, 1994. Reliability and stability of adult attachment patterns. Personal Relationships, 1: 23-43.

DOI: $10.1111 /$ j.1475-6811.1994.tb00053

Scott, K.A., S.J. Melhorn and R.R. Sakai, 2012. Effects of chronic social stress on obesity. Current Obesity Report, 1: 16-25. DOI: 10.1007/s13679-011-0006-3

Sobel, M.E., 1982. Asymptotic Confidence Intervals for Indirect Effects in Structural Equation Models. In: Sociological Methodology, Leinhardt, S. (Ed.), Jossey-Bass, San Francisco, ISBN-10: 0875894909, pp: 290-312.

Strategic Advantages, 2000. Symptom Assessment-45 Questionnaire (SA-45). Multi-Health Systems, New York.

Stroebe, W., E.K. Papies and H. Aarts, 2008. From homeostatic to hedonic theories of eating: Selfregulatory failure in food-rich environments. Applied Psychol. Int. Rev., 57: 172-193. DOI: $10.1111 /$ j.1464-0597.2008.00360

Stunkard, A. and S. Messick, 1985. The three-factor eating questionnaire to measure dietary restraint, disinhibition and hunger. J. Psychosomatic Res., 29: 71-83. DOI: 10.1016/0022-3999(85)90010-8

Cohen, J., 1988. Statistical Power Analysis for the Behavioral Sciences. 1st Edn., Lawrence Erlbaum, ISBN-10: 0805802835, pp: 567.

Suldo, S.M. and D.A. Sandberg, 2000. Relationship between attachment styles and eating disorder symptomatology among college women. J. College Student Psychotherapy, 15: 59-73. DOI: 0.1300/J035v15n01_07

Van Strien, T., R.C.M.E. Engels, J. Van Leeuwe and H.M. Snoeck, 2005. The Stice model of overeating: Tests in clinical and non-clinical samples. Appetite, 45: 305-213. DOI: 10.1016/j.appet.2005.08.004

WHO, 2013. Obesity and overweight fact sheet. World Health Organisation. 